ISSN: $1135-9250$

ED D T E c EUTEC. Revista Electrónica de Tecnología Educativa.

Número 60 / Junio 2017

\title{
USO DE LAS PLATAFORMAS EDUCATIVAS Y SU IMPACTO EN LA PRÁCTICA PEDAGÓGICA EN INSTITUCIONES DE EDUCACIÓN SUPERIOR DE SAN LUIS POTOSÍ
}

\section{USE OF PLATFORMS EDUCATIONAL AND ITS IMPACT ON TEACHING PRACTICE OF HIGHER EDUCATION INSTITUTIONS SAN LUIS POTOSI}

\author{
Wilmer Ramírez Valdez; wiravaslp@gmail.com \\ Centro de Altos Estudios Pedagógicos y Educativos de San Luis Potosí
}

Juan Ignacio Barajas Villarruel; willie@uaslp.mx

Facultad de Contaduría y Administración de la Universidad Autónoma de San Luis Potosí

\section{RESUMEN}

El propósito de la investigación fue determinar el impacto del uso de las plataformas educativas en la práctica pedagógica de nivel superior de San Luis Potosí. El estudio fue de tipo no experimental, con enfoque cuantitativo, correlacional y transversal. Se aplicó un cuestionario en línea a una población oculta de docentes para determinar el perfil de los participantes, el nivel de uso que hacen de las plataformas educativas y el impacto en su práctica pedagógica. Los resultados indicaron que los docentes perciben que entre más uso hacen de las plataformas educativas, el impacto en su práctica pedagógica es positivo. Asimismo, se evidenció una correlación significativa entre las categorías de planeación, desarrollo y evaluación, con su práctica docente.

PALABRAS CLAVE: Tecnología de la información, innovación educativa, ambiente de aprendizaje.

\section{ABSTRACT}

The purpose of the research was to determine the impact of the use of educational platforms in the higher education pedagogical practice of San Luis Potosí. The study was not experimental, with a quantitative, correlational and cross-sectional approach. An online questionnaire was applied to a hidden population of teachers to determine the profile of participants, the level of use they make of educational platforms and the impact on their pedagogical practice. The results indicated that teachers perceive that the more use they make of educational platforms, the impact on their pedagogical practice is positive. Likewise, there was a significant correlation between the planning, development and evaluation categories, with their teaching practice. 
KEYWORDS: Information Technology, Educational Innovation, Learning Environment.

\section{INTRODUCCIÓN}

Uno de los conceptos actuales es el de las Tecnologías de la Información y Comunicación (TIC). En la educación mexicana existen contrastes donde algunas escuelas cuentan con computadoras e internet y algunas que carecen de ellos. En el nivel superior a esa disparidad se agrega el hecho de que los docentes no poseen un origen común, prefiriendo el conocimiento empresarial sobre la formación pedagógica. Desde finales del siglo pasado en México se abre la posibilidad de la educación en línea y, dentro de esta, el uso de las plataformas educativas. En este contexto, existen cuestionamientos de ¿Cómo el uso de plataformas educativas impacta en la práctica pedagógica? y ¿cómo hacen algunos docentes para usar eficientemente las plataformas educativas y de dónde provienen sus conocimientos?

De acuerdo a Silvio (2005), una plataforma educativa es un entorno de trabajo en línea donde se comparten recursos para trabajar a distancia o en forma semipresencial las cuales deben poseer unas herramientas mínimas para su funcionamiento, divididas en las siguientes categorías:

1. Herramientas de gestión de contenidos, que permiten al profesor publicar y distribuir los materiales del curso entre los alumnos.

2. Herramientas de comunicación y colaboración, como foros, salas de chat y mensajería interna del curso.

3. Herramientas de seguimiento y evaluación, donde se pueden diseñar exámenes, publicar tareas, generar informes de la actividad de cada alumno, retroalimentar al alumno sobre su desempeño.

4. Herramientas de administración, donde se crean los grupos, se acepta a los alumnos y se da privilegios (permisos).

5. Herramientas complementarias, como sistemas de búsquedas de contenidos del curso, agregar aplicaciones.

Existen diversas denominaciones al término plataforma educativa, muchas de ellas usadas indiscriminadamente sin ser iguales en su concepción. Esto genera confusión dado que varios autores y sitios de Internet identifican a una misma opción de plataforma con diferente categoría. Algunas de las muchas denominaciones con que se catalogan a las plataformas virtuales y que se encuentran en internet son: (a) Entorno de Aprendizaje Virtual (EAV/VLE), (b) Sistema de Gestión de Aprendizajes (SGA/LMS), (c) Sistema de Gestión de Cursos (SGC/CMS), (d) Entorno de Gestión de Aprendizajes (EGA/MLE), (e) Plataforma de Aprendizaje (PA/LP) y (f) Aula Virtual (AV). Para efecto de esta investigación se llamó plataforma educativa a cualquiera de estas denominaciones para evitar confusiones y conflictos en los términos.

\section{REVISIÓN DE LA LITERATURA}

Achilli (1986) indicó que práctica docente es el trabajo que el maestro desarrolla cotidianamente en determinadas y concretas condiciones sociales, históricas e institucionales, adquiriendo una significación tanto para la sociedad como para el propio maestro. Al ser una práctica muy compleja, Fierro (1999) la dividió en seis dimensiones para analizarlas a profundidad: (a) 
dimensión personal, (b) dimensión institucional, (c) dimensión interpersonal, (d) dimensión social, (e) dimensión didáctica y (f) dimensión valoral.

Para esta investigación se unirán las dimensiones personal, interpersonal (tomando solo a maestro-alumnos) y didáctica y se conocerán como relación o práctica pedagógica. Por lo anterior, el concepto base de práctica pedagógica considerado en este trabajo es el que planteó Achilli (1986): proceso que se desarrolla en el contexto del aula en el que se pone de manifiesto una determinada relación maestro- conocimiento- alumno, centrada en el "enseñar" y el "aprender" (p. 6).

Porlán (1996), mencionó que un modelo pedagógico es una muestra de las alternativas posibles de enseñanza-aprendizaje. Para efecto de determinar el tipo de enseñanza que se efectúa al usar las plataformas educativas en esta investigación se consideró la propuesta de SCOPEO (2011), un organismo de la Universidad de Salamanca, el cual establece una clasificación tomando como base modelos de Mason (1998) y García (2004):

1. Modelo transmisivo. Los alumnos son receptores pasivos de la información. Lo que preocupa es el producto, sin tener en cuenta el proceso formativo

2. Modelo de transición. Lo importante son los intereses de los estudiantes, los procedimientos frente a los contenidos, el rol del profesor evoluciona hasta el de orientador y dinamizador de las experiencias de la clase.

3. Modelo integrador. Incluye diferentes perspectivas actuales y vigentes, como la constructivista e investigadora. Este enfoque acentúa la importancia de la adquisición de competencias específicas de naturaleza cognitiva, metacognitiva y social.

\subsection{Investigaciones sobre el tema}

Existen varias investigaciones sobre la tecnología en la enseñanza. Riccio y Gramacho (2004) realizaron una investigación sobre el uso de plataformas tecnológicas en cursos presenciales que se impartieron durante cuatro años a nivel licenciatura, donde afirmaron que los resultados de la utilización de las nuevas tecnologías en ambientes educacionales se vuelven más evidentes pasados algunos años de su introducción.

En el Instituto Tecnológico de Sonora, Cuevas, García y Cruz (2008), realizaron una investigación sobre el impacto de una plataforma para la gestión del aprendizaje utilizada en cursos presenciales. Asimismo, Prados, Boada, Poch y Soler (2004), analizaron la finalidad de las plataformas tecnológicas y llegaron a la conclusión de que, en su gran mayoría, son usadas para publicar material docente o bien para comunicarse con los alumnos a través de correo electrónico o foros. Desde el punto de vista de los autores, utilizar las plataformas tecnológicas para estos dos únicos fines es desaprovechar las posibilidades que ofrecen estos nuevos entornos de aprendizaje.

Fariña, González y Area (2013) investigaron el qué uso hacen de las aulas virtuales los docentes universitarios. Villamizar, Daza, Gómez, Archila, Ghisays y Rodríguez (2012) en su investigación 
sobre el uso actual del moodle como un soporte activo en los cursos de la Universidad del Rosario, identifican y describen el uso que le dan al moodle alumnos y profesores y determinan su impacto en el primer semestre del año 2012.

Salado (2012) elabora un análisis del impacto académico de la implementación de la plataforma moodle en el CESUES tanto para docentes como para alumnos. Saez (2012) realizó un estudio sobre la práctica pedagógica de las TIC y su relación con los enfoques constructivistas donde menciona como respuestas de los profesores la formación del profesorado, la necesidad de recursos materiales, la falta de tiempo para aplicar las TIC, la necesidad de aprovechar las ventajas de las TIC contra los beneficios pedagógicos de las TIC como enlace para seguir un modelo constructivista en busca de la autonomía y la colaboración.

Rodríguez y Álvarez (2013) realizaron una investigación sobre el análisis didáctico del aula virtual donde obtuvieron que predominan las actividades individuales, la evaluación de resultados sobre el proceso y donde los docentes reproducen una metodología transmisiva.

Basado en algunas de las investigaciones mencionadas anteriormente se propuso para este estudio los siguientes niveles de uso de acuerdo al conocimiento que deben de tener los docentes para utilizar herramientas. Estos niveles ayudan a fundamentar el impacto que pueden tener las plataformas educativas en la práctica pedagógica y se pueden clasificar en nivel alto (generar informes, seguimiento del proceso de aprendizaje), nivel medio (evaluar, gestionar servicios de comunicación y actividades colaborativas) y nivel bajo (distribuir material, gestionar grupos, cursos y actividades individuales).

\subsection{Pregunta de investigación}

La pregunta en la cual giró esta investigación fue: ¿cuál es el impacto del uso de las plataformas educativas en la práctica pedagógica en Instituciones de Educación Superior?

También se incluyeron preguntas complementarias como los son: ¿cuál es el perfil que tienen los docentes encuestados?, ¿cuál es el nivel de práctica pedagógica mediada por plataformas educativas en IES?, ¿cuál es el nivel de uso de las plataformas educativas por docentes de IES? y ¿qué factores del perfil docente influyen en el nivel de uso e impacto de las plataformas educativas?

\subsection{Propósito del estudio}

El propósito de este estudio fue determinar el impacto del uso de las plataformas educativas en la práctica pedagógica en Instituciones de Educación Superior de San Luis Potosí.

\subsection{Hipótesis del estudio}

En esta investigación se trató de comprobar la siguiente hipótesis: "El uso de las plataformas educativas provoca un impacto positivo en la práctica pedagógica en Instituciones de Educación Superior". 


\section{METOdOLOGÍA}

El diseño del estudio, fue no experimental, ya que se realiza sin manipular deliberadamente las variables, es decir, donde no hacemos variar intencionalmente las variables independientes (Hernández, Fernández y Baptista 2010). Tuvo un enfoque cuantitativo que, de acuerdo a los mismos autores, utiliza la recolección y el análisis de datos para contestar a la pregunta o preguntas de investigación y probar hipótesis establecida previamente, confía en la medición numérica, el conteo y el uso frecuente de estadística para establecer con exactitud patrones de comportamiento en una población.

El tipo de estudio fue correlacional, de acuerdo al alcance. En este tipo de estudio se pretende determinar el grado en el cual las variaciones en uno o varios factores son concomitantes con la variación en otro u otros factores (Tamayo, 2003). De acuerdo al tiempo en que se realiza la investigación fue transversal, ya que se pretende recoger datos de las unidades de análisis en un solo momento, en un tiempo único. Su propósito es describir variables y analizar su incidencia e interrelación en un momento dado (Hernández, Fernández y Baptista, 2010).

En este estudio se utilizó como variable independiente el uso de las plataformas educativas y como dependiente la práctica pedagógica mediante tecnología. También se usan dos variables para identificar el perfil sociodemográfico y el uso de las plataformas educativas del docente que realiza el estudio.

En la variable llamada perfil sociodemográfico se incluyeron los indicadores sexo, edad, escolaridad, institución, contrato, experiencia laboral y experiencia docente. La variable antecedentes en el uso de las plataformas educativas incluye los indicadores tipo de plataforma usada actualmente, capacitación tecnológica, capacitación en diseño instruccional, experiencia en plataformas educativas, número de materias apoyadas en plataformas educativas, número de veces a la semana que ingresa a la plataforma educativa y capacitación pedagógica.

En la variable práctica pedagógica mediante plataformas educativas se incluyen tres dimensiones planeación, desarrollo y evaluación. La dimensión planeación incluye los indicadores adaptación a la plataforma educativa, perfil y tipo de aprendizaje, objetivos didácticos, planificación coordinada de actividades, diseño de actividades, diversidad de actividades, actualidad de contenidos, aprovechamiento de la plataforma educativa, equilibrio de actividades, autonomía del alumno, practicidad de actividades y trabajo colaborativo. La dimensión desarrollo incluye los indicadores motivación inicial, claridad en instrucciones, clima entre participantes, diversidad de recursos didácticos, bibliografía, contenido extra y comunicación maestro - alumno. La dimensión evaluación incluye los indicadores diagnóstico, diversidad en evaluación, seguimiento del alumno, presentación de resultados y reflexión del proceso.

La variable uso de plataformas educativas tiene dos dimensiones: características e impacto. La dimensión características incluye los indicadores distribuir material, actividades individuales, evaluación, foros de discusión, gestionar comunicación, actividades colaborativas, generar informes y seguimiento del proceso de aprendizaje. La dimensión impacto tiene los indicadores planeación de clase, logro de objetivos, distribución de tiempo, gestión de la materia, clima del aula, participación, evaluación y aprendizaje de los alumnos. 
El instrumento de recopilación fue un cuestionario que fue estructurado por cinco secciones. La primera se enfocó en los datos generales. La segunda sección fue antecedentes en el uso de las Plataformas Educativas. La tercera sección se denominó práctica pedagógica del docente mediante el uso de las Plataformas educativas con un total de 25 preguntas usando escala Likert (siempre, casi siempre, algunas veces, rara vez y nunca), dentro de esta sección se incluyeron las dimensiones de planeación, desarrollo y evaluación. La cuarta sección se identificó como nivel de uso de las Plataformas educativas con un total de ocho preguntas usando escala Likert (siempre, casi siempre, algunas veces, rara vez y nunca). Por último, la sección de impacto de las Plataformas educativas con un total de ocho preguntas usando escala Likert (muy negativa, negativa, ni negativa ni positiva, positiva y muy positiva).

Se determinó la validación interna cuestionario con la aplicación de una prueba piloto con la participación de 36 profesores que no formaron parte de la muestra definitiva del estudio. Posteriormente se procesaron los resultados en el software SPSS donde se obtuvo un alfa de Cronbach general de .927. Al analizar cada pregunta el alfa rondó entre .921 la menor y .930 la mayor. En cuanto a la validación externa se entregó el cuestionario a tres expertos del área de investigación a nivel superior los cuales revisaron y aprobaron el instrumento.

Se solicitó apoyo a ocho instituciones de educación superior de la ciudad de San Luis Potosí para poder contactar a sus docentes y poderles aplicar el cuestionario. Solamente dos de ellas brindaron el apoyo solicitado, otras de ellas permitieron que se publicara en periódicos murales de maestros y el resto no brindaron apoyo, por lo que no se pudo calcular una muestra numérica mínima de docentes a encuestar.

Al ser así se optó por abrir la participación de los docentes mediante el uso de correo electrónico y la publicación de invitaciones a participar en la investigación mediante grupos de Facebook, Linkedin, Twitter y Edmodo.

El universo se definió como los docentes de Instituciones de Educación Superior de San Luis Potosí que manejan actualmente una plataforma educativa para sus asignaturas. La población está formada por los docentes de dichas instituciones que deseen participar en la encuesta en línea. Los criterios de inclusión fueron los docentes que laboren en IES y actualmente usen una plataforma educativa propia de la institución o gratuita de la red como apoyo para sus asignaturas. Los criterios de eliminación fueron docentes que hayan muerto, dados de baja, o que decidan no participar en la investigación.

Se propuso el tiempo del nueve de noviembre al diez de diciembre de 2015 para convocar a los docentes para participar en la investigación. Al no poderse calcular una muestra y llegar a todos los docentes que se pretendía, se buscó otra alternativa, esta fue el usar una población oculta. Se denomina población oculta ya que es de difícil reconocimiento y estimación, no se puede obtener una muestra pues se desconoce el total de la población (Wiebel, 1990).

El muestreo elegido fue no probabilístico porque se desconoce la probabilidad de seleccionar cualquier miembro individual de la población y se realizó a través de dos técnicas: por cuotas y bola de nieve. En el caso de este estudio al usar redes sociales y correos electrónicos se orientó la muestra a los docentes que usan internet y que, por tanto, tienen mayor posibilidad de usar plataformas educativas. También se adaptó esta técnica para que la persona interesada compartiera el enlace con sus conocidos, los cuales cumplen con las mismas características del sujeto, por lo que la muestra se mantiene intacta. Se es consciente que al usar dichas técnicas de 
muestreo no se tiene certeza de la representatividad de la muestra ni del grado de confianza obtenido ya que es difícil calcular el porcentaje alcanzado.

Al finalizar el tiempo se habían recibido 128 respuestas. Se procedió a exportar las respuestas de Google Drive a SPSS 23, donde se procedió a realizar estadísticas por ítem, por bloque y a nivel general. Las pruebas estadísticas realizadas fueron de frecuencia, se calcularon posteriormente nuevas variables en base a la suma de indicadores por dimensión de la variable práctica pedagógica (planeación, desarrollo y evaluación) que se usaron para obtener la correlación bivariada, y regresión lineal entre práctica pedagógica e impacto y entre características e impacto. Por último, se obtuvieron tablas cruzadas entre las respuestas de cada pregunta de las variables perfil sociodemográfico, antecedentes, práctica pedagógica y uso de plataformas educativas dimensión características contra nivel de impacto.

\section{RESULTADOS}

De los resultados obtenidos se pueden destacar los siguientes:

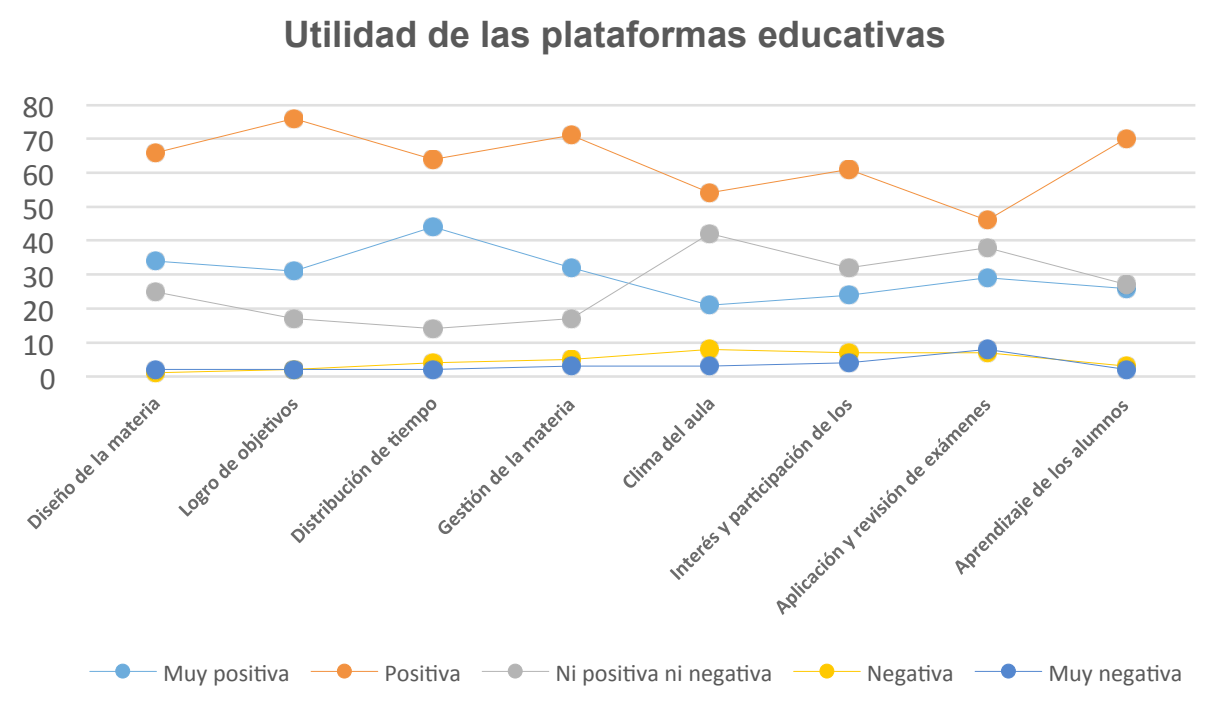

Figura 1: Comparación utilidad de las plataformas educativas

Los docentes consideran que la utilidad de la plataforma en los ocho aspectos manejados en la encuesta (diseño de la materia, logro de objetivos, distribución de tiempo, gestión de la materia, clima del aula, interés y participación de los alumnos, aplicación y revisión de exámenes, aprendizaje de los alumnos) es positiva o muy positiva. Sobresale el aprendizaje de los alumnos con el $75 \%$.

Todo esto apoya los resultados de Salado (2012) que, en su investigación sobre el análisis del impacto académico de la implementación de la plataforma Moodle en el CESUES, muestra que el 92\% de los profesores consideraron que la utilización de la Plataforma institucional ha facilitado el 
contacto con los alumnos, mayor dedicación al preparar clase, genera mayor actitud positiva en el desarrollo académico.

También apoya a Pérez, Aguaded y Fandos (2010) que, dentro de su investigación el tratamiento de la información y la competencia lingüística en los Centros TIC andaluces, encontraron que los docentes señalaron que sus clases se vuelven más activas y participativas facilitando el aprendizaje autónomo de su alumnado, y adquiriendo nuevos conocimientos a través de trabajos de investigación en los que el docente pasa a ser un guía en el aprendizaje de sus alumnos y alumnas. Así también en el aula se señalan mejoras en la participación del alumnado, trabajo autónomo, y dinámica del grupo. En menor grado se señalan mejoras en la comunicación profesoradoalumnado, alumnado-alumnado, y en el clima del aula.

Tabla cruzada nivel uso*nivel impacto

\begin{tabular}{cccccc}
\hline \multirow{5}{*}{ nivel uso } & & \multicolumn{4}{c}{ nivel impacto } \\
\cline { 3 - 6 } & Alto & $56.1 \%$ & $22.6 \%$ & $0.0 \%$ & $38.3 \%$ \\
& & & & \\
Medio & $27.3 \%$ & $39.6 \%$ & $11.1 \%$ & $31.3 \%$ \\
Bajo & $16.7 \%$ & $37.7 \%$ & $88.9 \%$ & $30.5 \%$ \\
Total & $100.0 \%$ & $100.0 \%$ & $100.0 \%$ & $100.0 \%$ \\
\hline \multicolumn{4}{c}{ Tabla 1 } & &
\end{tabular}

La tabla 1 muestra un cruce de datos entre nivel de uso y nivel de impacto para identificar cómo perciben el impacto los docentes al usar más las diferentes herramientas de las plataformas educativas. Se puede observar que mientras mayor es el nivel de uso de la plataforma educativa más impacto positivo encuentran los docentes en su práctica pedagógica (56.1\%) y que mientras menor es el nivel de uso mayor es el impacto negativo encontrado (88.9\%).

Usos de las plataformas educativas

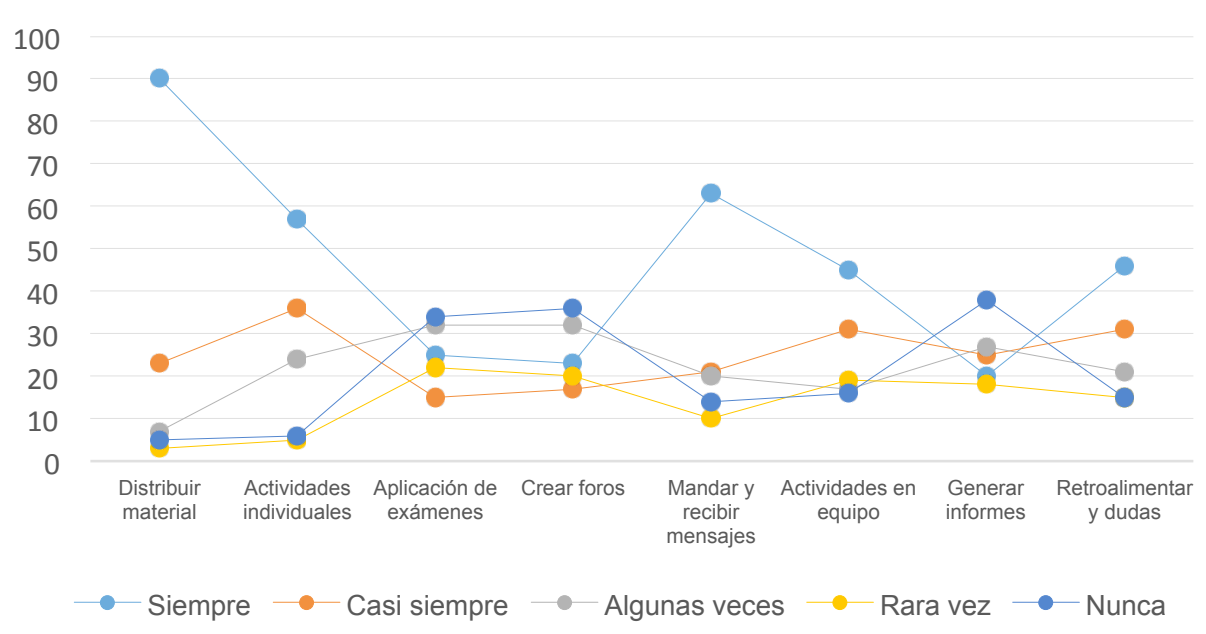

Figura 2: Comparación del uso de las plataformas educativas 
Se obtuvo que los docentes perciben como herramientas principales y de mayor uso a la distribución de material, encargar actividades individuales, mandar y recibir mensajes, encargar actividades en equipo y retroalimentar y responder dudas; solamente la aplicación de exámenes $(31.2 \%)$, crear foros (31.3\%) y generación de informes (35.1\%) no se utilizan en un nivel alto.

De la misma manera, más de la tercera parte de los docentes (36.2\%) indican que usan las plataformas educativas solo para distribuir material y encargar actividades individuales (nivel bajo). Un $42.38 \%$ indican que la usan para aplicar exámenes, crear foros, enviar y recibir mensajes y encargar actividades en equipos además del nivel anterior (nivel medio de uso); y un $21.42 \%$ indican que la usan para generar informes y retroalimentar a los alumnos y los dos niveles anteriores (nivel alto de uso).

Correlación Impacto / Planeación, Desarrollo, Evaluación, Uso

\begin{tabular}{llllll}
\hline \multirow{2}{*}{$\begin{array}{l}\text { nivel } \\
\text { impacto }\end{array}$} & $\begin{array}{l}\text { Correlación de } \\
\text { planeación }\end{array}$ & $\begin{array}{l}\text { nivel } \\
\text { desarrollo }\end{array}$ & $\begin{array}{l}\text { nivel } \\
\text { evaluación }\end{array}$ & $\begin{array}{l}\text { nivel } \\
\text { uso }\end{array}$ \\
\cline { 3 - 6 } & Pearson & $.419^{* *}$ & $.486^{* *}$ & $.402^{* *}$ & $.449^{* *}$ \\
& Sig. (bilateral) & .000 & .000 & .000 & .000 \\
& $\mathrm{~N}$ & 128 & 128 & 128 & 128 \\
\hline
\end{tabular}

Tabla 2 :La correlación es significativa en el nivel 0,01 (bilateral)

En la tabla 2 se puede observar la correlación entre impacto y las 4 categorías de las preguntas. Todas, planeación .419, desarrollo .486, evaluación .402 y uso .449 tienen una correlación directa (positiva) con el impacto, que si bien no es fuerte si es significativa. Juntas, estas explican el $28.8 \%$ de la variación del impacto.

Tabla cruzada Nivel de estudios*nivel impacto

\begin{tabular}{llrrrr}
\hline & \multicolumn{5}{c}{ nivel impacto } \\
& & positivo & neutro & negativo & Total \\
\cline { 3 - 6 } Nivel de estudios & Licenciatura & $19.7 \%$ & $18.9 \%$ & $0.0 \%$ & $18.0 \%$ \\
& Maestría & $56.1 \%$ & $41.5 \%$ & $22.2 \%$ & $47.7 \%$ \\
Total & Doctorado & $24.2 \%$ & $39.6 \%$ & $77.8 \%$ & $34.4 \%$ \\
& & $100.0 \%$ & $100.0 \%$ & $100.0 \%$ & $100.0 \%$ \\
\hline
\end{tabular}

Tabla 3

En la tabla 3 se observa el nivel de impacto por nivel de estudios. En el caso de la opinión del impacto positivo de las plataformas educativas $19.7 \%$ poseen licenciatura, $56.1 \%$ maestría y $24.2 \%$ doctorado. Del impacto neutro $18.9 \%$ poseen licenciatura, $41.5 \%$ maestría y $39.6 \%$ doctorado. Del impacto negativo $22.2 \%$ poseen maestría, $77.8 \%$ doctorado y con nivel licenciatura no hay opiniones. 
También se obtuvo que los docentes perciben que planean mediante la plataforma educativa en un nivel alto, pero el aspecto de coordinarse con otros docentes de la materia en academias no está muy difundido aún ya que solo el $31.2 \%$ de los docentes indicaron que planean actividades y evaluaciones de este modo. Que perciben que desarrollan sus clases mediante la plataforma educativa en un nivel alto a través de indicar las instrucciones de las actividades, distribuir material y comunicarse con los alumnos. Y que perciben que evalúan mediante la plataforma en un nivel alto al usar diferentes herramientas para evaluar actividades, retroalimentar a los alumnos e informar oportunamente a los alumnos de sus calificaciones. Aspectos a trabajar son la evaluación diagnóstica (28.2\%) y la autoevaluación de la estrategia didáctica (38.3\%) que no son muy usadas por los docentes.

De acuerdo al promedio general de las diferentes categorías en los 25 indicadores, un poco más de la mitad de los docentes (56.19\%) perciben que tienen un nivel alto de práctica pedagógica mediada por tecnología por lo que de acuerdo a SCOPEO (2011) el modelo implementado por ellos es integrador (permite trabajar competencias y brinda autonomía al alumno a través de la comunicación y la investigación). Destacar que solo en el $64 \%$ de los indicadores la práctica pedagógica alta supero el $50 \%$.

La mayoría de los participantes laboran en instituciones públicas. Al comparar las respuestas de los participantes de este tipo de instituciones contra los de instituciones privadas se observa que concentran el mayor porcentaje de nivel de uso bajo (97.4\%) e impacto negativo (100\%). También concentran el mayor porcentaje que no ha tomado capacitación sobre uso de la plataforma (96\%), capacitación sobre diseño instruccional (96.4\%) y capacitación pedagógica (100\%). Por último, concentran el mayor porcentaje de nivel bajo en planeación (100\%), desarrollo (95\%), evaluación $(98.1 \%)$ y uso $(97.4 \%)$.

\section{CONCLUSIONES}

A partir de los resultados del estudio, recordando que se obtienen a partir de las percepciones de los docentes, se puede concluir que la mayoría de los docentes encuentran un impacto positivo de las plataformas educativas, seguido de un impacto neutro. Menos de la décima parte de los docentes indicaron un impacto negativo. Aceptando así la hipótesis inicial del estudio "El uso de las plataformas educativas provoca un impacto positivo en la práctica pedagógica en IES".

Una conclusión más es sobre los perfiles docentes que usan en un nivel alto y bajo las plataformas educativas. El perfil docente que usa en un nivel alto es de género femenino, posee entre 41 a 50 años, entre 10 y 19 años de experiencia laboral, menos de 10 años de experiencia docente, cuenta con maestría, usa Moodle, ha usado plataformas educativas por más de tres años y está capacitado en los tres cursos. El perfil docente que usa en un nivel bajo las plataformas educativas es de género masculino, posee entre 51 y 60 años, menos de 10 años de experiencia laboral y docente, cuenta con doctorado, usa Moodle, ha usado plataformas educativas por más de tres años, ha tomado capacitación sobre el uso de la plataforma educativa y capacitación pedagógica.

Otra conclusión es sobre los perfiles docentes que perciben un impacto positivo y negativo de las plataformas educativas. El perfil docente que percibe un impacto positivo de las plataformas educativas es de género masculino, posee entre 41 a 50 años, entre 10 y 19 años de experiencia 
laboral, menos de 10 años de docente, cuenta con maestría, usa Moodle, ha usado plataformas educativas por más de tres años y está capacitado en los tres cursos. El perfil docente que percibe un impacto negativo de las plataformas educativas es de género femenino, posee de 51 a 60 años, menos de 20 años de experiencia laboral, menos de 10 años de experiencia docente, cuenta con doctorado, usa Moodle, menos de un año y más de tres años usando plataformas educativas, ha tomado capacitación sobre el uso de la plataforma educativa.

También se puede concluir que la capacitación sobre diseño instruccional es la que genera mayor nivel de uso, impacto positivo y disminuye el nivel de uso bajo y el impacto negativo. La capacitación del diseño instruccional y pedagógica forman una buena combinación ya que generan una mayor percepción del impacto positivo de las plataformas educativas así como una menor percepción del impacto negativo de estas.

Por último, se puede concluir que de acuerdo a lo que indicaron los docentes en el estudio un nivel alto de las categorías de planeación, desarrollo y evaluación provoca un nivel alto de uso. En el caso del nivel de uso bajo es provocado por un nivel bajo de desarrollo y evaluación, pero no de planeación, donde se genera por un nivel medio de esta categoría.

\section{RECOMENDACIONES}

Se recomienda para futuras investigaciones la participación extendida de las instituciones de nivel superior brindando la información con la cual contactar a sus docentes para que pueda llegar a más personas y obtener resultados generalizables.

También se recomienda utilizar otros instrumentos de recopilación de información como observación, entrevistas o grupos focales para proporcionar una mirada más detallada de la práctica pedagógica mediante plataformas educativas en instituciones de nivel superior dado que los resultados fueron obtenidos directamente de cuestionarios a los docentes por lo que proceden de su percepción del uso e impacto de las plataformas educativas.

\section{REFERENCIAS}

ACHILLI, E. (1986). La práctica docente: Una interpretación desde los saberes del maestro. Cuadernos de formación docente, Universidad Nacional de Rosario.

ATKINSON, R. y FLINT, J, (2001), Accessing Hidden and Hard-to-Reach Populations: Snowball Research Strategies, Social Research Update, University of Surrey, 33: 1-5.

BOZA, Á., TIRADO, RAMÓN \& GUZMÁN-FRANCO, MARÍA-DOLORES (2010). Creencias del profesorado sobre el significado de la tecnología en la enseñanza: influencia para su inserción en los centros docentes andaluces. RELIEVE, vol. 16, n. 1, p. 1-24. http://www.uv.es/RELIEVE/v16n1/RELIEVEv16n1_5.htm 
CUEVAS S., GARCÍA L. \& CRUZ M. (2008). Evaluación del impacto de una plataforma para la gestión del aprendizaje utilizada en cursos presenciales en el ITSON, Revista Mexicana de Investigación Educativa, RMIE, octubre-diciembre 2008, VOL. 13, NUM. 39. PP. 1085-1107.

FARIÑA, E.; GONZÁLEZ, C.S. y AREA, M. (2013). ¿Qué uso hacen de las aulas virtuales los docentes universitarios? RED, Revista de Educación a Distancia. Número 35. 1 de Enero de 2013. Consultado el (19 de febrero de 2014) en http://www.um.es/ead/red/35/

FIERRO C. (1999). Transformando la práctica docente. Argentina: Paidós.

GARCÍA ARETIO, L. (2004). Algunos modelos de Educación a Distancia, en García, L. (2009) ¿Por qué va ganando la Educación a distancia? Madrid, UNED.

HERNÁNDEZ, R., FERNÁNDEZ, C. \& BAPTISTA, P. (2010). Metodología de la investigación. Distrito Federal, México: McGraw-Hill.

MASON, R. (1998). Models of Online Courses. ALN Magazine, 2 (2). Extraído el 24 de febrero de 2015

http://wwwusers.york.ac.uk/ ijc4/etutoring/week\%201/Robin\%20Mason\%20paper.doc

PÉREZ R.; AGUADED, J. \& FANDOS, M. (2010). El Tratamiento de la Información y la Competencia Lingüística en los Centros TIC Andaluces, Meta: Evaluación| Río de Janeiro, vol. 2, n. 6, p. 358-400, sep/dic 2010.

PORLÁN, R. (1996). Cambiar la Escuela. Buenos Aires, Argentina: Magisterio del Rio de la Plata.

PRADOS, R; BOADA, I.; POCH, J. \& SOLER, J. (2004). El e-learning como complemento a las clases presenciales. Un caso práctico: el proyecto ACME, trabajo presentado en el Congreso Virtual Educa 2004, del 16 al 18 de julio, disponible en http://acme.udg.es/explicacio/acme.php?opcio=4.

RICCIO, E. L. \& GRAMACHO, M. C. (2004). Estrategias innovadoras en la enseñanza superiorexperiencias y percepciones resultantes del uso de Internet y webCT en TECSI/ FEA/USP, Revista Electrónica Arbitrada, 1(2) julio, pp. 1-15, disponible en http://www.tecsi.fea. usp.br/riccio/artigos/pdf/Riccio\&Sakata-APCAM2vl-2004.pdf

RODRÍGUEZ, C. \& ÁLVAREZ, M. J. (2013). Análisis didáctico de las aulas virtuales. Una investigación en un contexto de educación superior. EDUTEC, Revista Electrónica de Tecnología Educativa, $44 . \quad$ Recuperado el 29/06/16 de http://edutec.rediris.es/Revelec2/Revelec44/analisis_didactico_aulas_virtuales_educacion _ superior.html

SÁEZ, J. (2012). La práctica pedagógica de las tecnologías de la información y la comunicación y su relación con los enfoques constructivistas. Revista Iberoamericana sobre calidad, eficacia y cambio en educación. Volumen 10, número 1. 
SALADO L. (2012). Análisis del impacto académico de la implementación de la plataforma moodle en el CESUES. Revista internacional Administración \& Finanzas. Volumen 5, número 2. Disponible en http://www.theibfr2.com/RePEc/ibf/riafin/riaf-v5n2-2012/RIAF-V5N2-20128.pdf

SCOPEO (2011). Aproximación pedagógica a las plataformas open source en la universidad española. Universidad de Salamanca.

SILVIO, J. (2005). Sense of community, perceived cognitive learning, and persistence in asynchronous learning networks. The Internet and Higher Education, 5 (4), 319-332

TAMAYO, M. (2003). El proceso de investigación científica. México: Limusa.

VILLAMIZAR, C.; DAZA, S.; GÓMEZ, C.; ARCHILA, H.; GHISAYS, R. \& RODRÍGUEZ, J. (2012). El uso actual del moodle como un soporte activo en los cursos de la Universidad del Rosario, Universidad del rosario, Bogotá.

WIEBEL, W. (1990) Identifying and Gaining Access to Hidden Populations In: Elizabeth Y. Lambert (Ed.): The Collection and Interpretation of Data from Hidden Populations. National Institute on Drug Abuse. Research Monograph Series. 98. DHSS publication number (ADM) 90-1678 Rockville, MD. pp 4-13

\section{Para citar este artículo:}

RAMÍREZ, W. \& BARAJAS, J.I. (2017). Uso de las plataformas educativas y su impacto en la práctica pedagógica en instituciones de educación superior de san luis potosí. EDUTEC, Revista Electrónica de Tecnología Educativa, 60. Recuperado el dd/mm/aa de http://www.edutec.es/revista 